\title{
Quand l'exceptionnel dure et le provisoire se normalise : les Centres d'Accueil et d'Orientation pour mineurs non accompagnés (CAOMI)
}

As Exceptional Lasts and Temporory Tends to Become Normal: Unaccompanied Minors Asylum Reception Centers (CAOMI)

Cuando lo excepcional perdura y lo provisional se normaliza: los centros de acogida y de orientación para menores no acompañados (CAOMI)

\section{Florian Aumond}

\section{(2) OpenEdition}

\section{Journals}

Édition électronique

URL : https://journals.openedition.org/remi/8505

DOI : $10.4000 /$ remi.8505

ISSN : $1777-5418$

Éditeur

Université de Poitiers

Édition imprimée

Date de publication : 1 mars 2017

Pagination : 157-163

ISBN : 979-10-90426-30-6

ISSN : 0765-0752

\section{Référence électronique}

Florian Aumond, «Quand l'exceptionnel dure et le provisoire se normalise : les Centres d'Accueil et d'Orientation pour mineurs non accompagnés (CAOMI) », Revue européenne des migrations internationales [En ligne], vol. $33-n^{\circ} 1$ | 2017, mis en ligne le 01 mars 2019, consulté le 14 avril 2022 URL : http://journals.openedition.org/remi/8505 ; DOI : https://doi.org/10.4000/remi.8505 


\section{Chronique juridique}

\section{Quand l'exceptionnel dure et le provisoire se normalise : les Centres d'Accueil et d'Orientation pour mineurs non accompagnés (CAOMI)}

\section{Florian Aumond ${ }^{1}$}

Les temps qualifiés d'exceptionnels sont propices à la mise en place d'un droit dérogatoire, droit de l'exception. Ainsi, de la menace terroriste qui pèserait avec une particulière acuité sur la France et justifierait l'instauration d'un état d'urgence dont on ne sait trop quand, voire s'il s'achèvera et dont une partie du dispositif a en tout état de cause déjà impacté le droit commun. Ainsi également - et le lien avec l'exemple précédent est assurément peu heureux, même si fréquemment esquissé -, de ladite " crise migratoire " (Collectif, 2016). L'afflux qualifié de " massif $"{ }^{\otimes}$ de migrants commanderait d'adapter la législation et les dispositifs prévalant en temps "normal ». Mais l'on peut craindre, comme pour l'état d'urgence, qu'en la matière aussi, l'exceptionnel dure et le provisoire ne se normalise. On en veut pour preuve le dispositif institué dans le cadre du démantèlement de ladite " jungle " de Calais et la mise en place des Centres d'Accueil et d'Orientation pour mineurs non accompagnés (CAOMI)².

La question de la présence en France de mineurs privés temporairement ou définitivement d'une protection parentale ne s'est véritablement posée que récemment ${ }^{3}$. Le nouveau développement du phénomène dans les années 2000 a interrogé la répartition des rôles, concernant leur prise en charge, entre l'État et les départements. Or, par-delà la simple organisation territoriale de la République, le choix ici à opérer renvoie plus profondément au biais avec lequel I'on choisit d'aborder la situation de ces jeunes caractérisés par leur minorité, leur isolement et leur extranéité (Senovilla Hernández, 2014). D’un côté, les deux

\footnotetext{
1 Maître de conférences en droit public, Université de Poitiers, CECOJI-UP/MIGRINTER, UFR Droit et sciences sociales, 15 rue Sainte-Opportune, Bât. E10, TSA 81100, 86073 Poitiers cedex 9 ; florian.aumond@univ-poitiers.fr

2 On relèvera avec intérêt le décalage entre la dénomination et I'abréviation, les " mineurs non accompagnés " devenant MIE. II y a là peut-être la manifestation d'une mutation dans la sémantique utilisée en droit français qui, jusqu'à présent et contrairement aux droits européen comme international, usait presque exclusivement de l'expression " mineurs isolés étrangers ". Par la suite, on conservera I'expression MIE, tout en conservant à l'esprit les évolutions qui semblent en cours.

3 Elle émerge avec l'arrivée au début des années 1980 de jeunes Yougoslaves, puis prend de l'ampleur au milieu des années 1990 avec des jeunes venant notamment du Maghreb (Debré, 2010).
} 
premiers éléments postulent l'intervention des départements, à qui compétence est dévolue, expressément depuis la loi du 5 mars 2007 réformant la protection de l'enfance, au titre de la protection de l'enfance. De l'autre, en tant qu'ils sont étrangers, ils pourraient également relever de la politique migratoire organisée par l'État.

Les relations entre États et collectivités territoriales se tendent au début des années 2010. Afin de signifier son refus de prendre à sa charge un nombre croissant de mineurs arrivant dans son département, le président du Conseil général de Seine-Saint-Denis met à exécution une menace précédemment brandie et suspend l'accueil de nouveaux mineurs (Cavaniol, 2013). Après d'âpres négociations, un dispositif de répartition nationale et de solidarité départementale est annoncé par le ministre de la Justice dans un discours prononcé devant l'Assemblée des maires de France (octobre 2011), avant d'être officialisé dans une circulaire relative aux modalités de prise en charge des mineurs isolés étrangers : dispositif national de mise à l'abri, d'évaluation et d'orientation, en date du 31 mai 2013. Le rappel des grandes lignes de ce mécanisme de droit commun permettra alors de mieux saisir les particularités de celui imaginé dans le contexte du démantèlement de Calais.

\section{Le dispositif de droit commun concernant la mise à I'abri, l'évaluation et l'orientation des MIE}

Le dispositif instauré dans la circulaire du 31 mai 2013 distingue pour l'essentiel deux phases. La première concerne la mise à l'abri et l'évaluation (ou accueil provisoire d'urgence). II revient ici au Conseil général ${ }^{4}$ dans le département duquel est repéré ou s'est présenté un jeune se déclarant mineur de l'héberger et de procéder à l'évaluation de sa situation. Une solidarité nationale est organisée dans ce premier temps, puisque son financement incombe à l'État, dans la limite cependant de cinq jours. Ce délai, court, vise à inciter le Conseil général (départemental) à procéder à une évaluation rapide. Dans I'hypothèse où la minorité comme l'isolement sont clairement établis durant cette période, il revient au président du Conseil général (départemental) de saisir le procureur de la République du lieu où le mineur a été trouvé. À défaut, il doit le solliciter pour que ce dernier le lui confie par une ordonnance de placement provisoire.

La conclusion de l'évaluation emporte basculement vers la seconde phase, celle de l'orientation en vue d'un placement définitif du mineur isolé étranger. La solidarité (verticale) nationale cède ici la place à une solidarité (horizontale) départementale. En effet, la prise en charge relève désormais du seul Conseil général (départemental) du lieu de ce placement définitif (par le biais de ses services d'aide sociale à l'enfance [ASE]), mais Conseil pouvant ne pas être celui ayant procédé à la phase initiale de mise à l'abri et d'évaluation. Le procureur en charge de le désigner doit pour ce faire s'appuyer, aux termes de la circulaire du 31 mai 2013, sur un dispositif national d'orientation institué afin d'éviter la concentration des MIE dans les départements où ils sont principalement localisés en début de processus (soit, principalement, au nord, en région parisienne et dans les Bouches-du-Rhône). Ce dispositif repose sur une clé de répar-

4 Désormais renommé Conseil départemental. 
tition et s'appuie sur l'intervention d'une cellule nationale placée à la direction de la protection judiciaire de la jeunesse (PJJ) (le placement définitif est le fait du juge des enfants ayant compétence dans le territoire où il est effectué).

II est peu de dire que ce mécanisme n'a pas résorbé toute difficulté ni éteint tout contentieux. Les réticences de certains Conseils généraux (départementaux) à accueillir des mineurs ont été portées devant les prétoires, soit par des mineurs ayant essuyé des refus, soit par les Conseils eux-mêmes, contestant devant le juge administratif le dispositif instauré par la circulaire (Domingo, 2015). Sur ce second point, après un premier rejet en référé (Conseil d'État [CE], ord. 10 mars 2014, Département de l'Aveyron et a.), un arrêt du 30 janvier 2015 vient apporter d'utiles précisions (CE, 30 janvier 2015, Département des Hauts-de-Seine). Le juge administratif fait sienne, d'une part, une critique formulée entre autres par la Commission nationale consultative des droits de l'homme (CNCDH) à I'encontre d'un dispositif de répartition prenant essentiellement en compte les enjeux financiers à l'exclusion de toute considération de l'intérêt supérieur de I'enfant (CNCDH, Avis sur la situation des MIE présents sur le territoire national, 27 juin 2014). Le Conseil d'État conteste en effet la référence faite dans la circulaire du 31 mai 2013 à " l'absence de critère législatif " devant orienter le choix, par le procureur, du département d'accueil définitif, en rappelant que la loi impose la prise en considération de l'intérêt supérieur de l'enfant (ISE) dans les décisions de placement définitif comme provisoire, y compris pour les MIE. À l'inverse, et là est le second apport de la décision du 30 janvier 2015, la loi ne prescrit aucunement au procureur de prendre en considération un critère tiré de la nécessité de limiter les disparités dans les flux d'arrivée des MIE. Le procureur n'est dès lors en rien lié par les informations recueillies auprès de la cellule nationale. Ces dernières conservent toutefois une utilité dans la mesure où, précise le Conseil d'État, il est dans l'intérêt supérieur de l'enfant d'être pris en charge par les Conseils généraux (départementaux) dans des conditions satisfaisantes. Ce qui ne serait donc pas le cas pour ceux considérés comme saturés.

La décision du Conseil d'État a été suivie d'effets dans sa double conclusion. Concernant la première, les difficultés étaient certes moindres puisqu'il n'y avait pas lieu de modifier l'état du droit positif, mais seulement d'en rappeler la teneur selon l'interprétation livrée par le Conseil d'État. Ce à quoi procède, dès le 17 février 2015, le ministre de la Justice par une nouvelle circulaire recommandant aux parquets, lorsqu'ils décident du placement provisoire ou définitif d'un MIE auprès d'un Conseil général (départemental), de se prononcer au regard du critère de I'ISE. La circulaire précise alors, utilement, que pour les MIE, la caractérisation de cet intérêt s'appuie sur un ensemble de critères, dont "son environnement, l'adéquation du lieu d'accueil avec ses besoins [ou] le maintien des liens avec ses frères et sœurs ". En revanche, la consécration d'un nouveau critère législatif d'orientation, fondé sur une répartition tendant vers une certaine équité entre départements, supposait par définition l'adoption d'une loi. Celle du 14 mars 2016, relative à la protection de I'enfance, en a fourni I'occasion. Elle introduit de fait un nouvel article L. 221-2-2 dans le Code de I'action sociale et des familles (CASF) prévoyant, en particulier, que " le ministre de la Justice fixe les objectifs de répartition proportionnée des accueils [des MIE] entre les départements, en fonction de critères démographiques et d'éloignement géographique " (si I'ISE n'est pas ici mentionné, il l'est en revanche dans la nouvelle rédaction de l'article 375-5 du Code civil, organisant entre autres les pouvoirs du 
procureur de la République et du juge des enfants dans I'orientation des MIE, issue de la même loi du 14 mars 2016). Les principes de répartition nationale et de solidarité départementale trouvent ainsi une base légale. Les modalités de leur application ont par la suite été précisées par un décret d'application du 24 juin 2016 - lui-même complété par un arrêté interministériel du 17 novembre 2016. Il en ressort une nouvelle clé de répartition passablement complexe définie à I'article R. 221-11 CASF.

Reste que, de nouveau, tout problème dans la répartition horizontale (entre départements) comme verticale (État/département) de la prise en charge des MIE ne se trouve pas de ce simple fait résolu. Sur le premier point, les difficultés, parfois réelles, rencontrées par certains départements ont ainsi motivé l'adoption d'une nouvelle circulaire interministérielle venant préciser, notamment dans le domaine de l'éducation, de la formation et de l'insertion professionnelles ou de la santé, "I'articulation entre les Conseils départementaux et les services de l'État dans leurs champs de compétence respectifs " (Circulaire interministérielle relative à la mobilisation des services de l'État auprès des Conseils départementaux concernant les mineurs privés temporairement ou définitivement de leurs familles ou présentés comme tels, 25 janvier 2016). En revanche, la quasiparalysie du dispositif de répartition nationale entre octobre 2015 et avril 2016 est largement imputable au refus systématique de nombre de départements de faire preuve de solidarité. Elle a eu pour conséquence le maintien de MIE dans le dispositif de l'accueil provisoire, affectant au premier chef ceux présents dans ladite " jungle » de Calais.

\section{Le dispositif de mise à l'abri, d'évaluation et d'orientation spécifique dans le cadre des opérations de démantèlement de Calais}

La situation à Calais et, plus largement dans le Calaisis, a concentré I'attention sur la situation actuelle des migrations, en France et, au-delà, en Europe. Elle a également donné lieu à une séquence (non refermée) sur le plan juridique. Des institutions nationales des droits de l'homme (INDH) : Défenseur des droits (DD), Commission nationale consultative des droits de l'homme (CNCDH) y ont joué un rôle de tout premier ordre dans la garantie du respect des droits des migrants, en général, des MIE, en particulier. D'autant que leur fonction d'alerte a trouvé un certain écho auprès du juge.

II en va particulièrement ainsi du rapport du Défenseur des droits intitulé Exilés et droits fondamentaux : la situation des exilés sur le territoire du Calaisis et publié en octobre 2015. Son influence sur l'ordonnance du Tribunal administratif de Lille (2 novembre 2015), confirmée sur ce point par le Conseil d'État (23 novembre 2015), rendue sur requêtes présentées par des associations au sujet de la situation prévalant dans le " bidonville de Calais ", est indéniable. Le juge administratif enjoint en effet au préfet de procéder au recensement des MIE présents dans la zone, ainsi que de " se rapprocher du département du Pas-deCalais en vue de leur placement ". Le Défenseur des droits se montre par la suite vigilant dans la mise en œuvre de cette double obligation reprenant ses préconisations ainsi, plus largement, que dans le traitement des MIE dans le Calaisis. 
Il diligente plusieurs visites sur place (notamment effectuées par la Défenseure des enfants, son adjointe) au cours desquelles est relevée une certaine incurie des autorités. L'ouverture à compter d'octobre 2015 des Centres d'Accueil et d'Orientation (CAO) sur l'ensemble du territoire, devant accueillir provisoirement les migrants ne souhaitant pas prolonger leur parcours vers le Royaume-Uni et le temps d'enclencher les démarches en vue notamment de se voir accorder une protection internationale, ne constitue ainsi pas une alternative satisfaisante pour les MIE. D'une part, la plupart maintiennent leur projet de se rendre outre-Manche, ce qui implique d'envisager une mise à l'abri sur place. $\mathrm{D}^{\prime}$ autre part, et pour les autres, ces CAO ne sont ainsi pas spécialement adaptés pour les mineurs. S'il obvie à la première critique, le Centre d'Accueil Provisoire (CAP) ouvert en janvier 2016 ne propose pas davantage une solution acceptable sur le long terme.

Les craintes du Défenseur de droits se trouvent ravivées à l'annonce du démantèlement de ladite " jungle " de Calais. La première phase, concernant la partie sud (février), fait l'objet de réserves appuyées dans une première décision rendue le 20 avril 2016 (MDE-2016-113). Il y déplore les obstacles persistants à la réalisation du dispositif d'orientation, en raison de l'obstruction de nombre de départements, qui ont pour effet de faire de l'accueil provisoire une solution pérenne et de principe. Le Défenseur des droits manifeste en outre son impatience au sujet des négociations entre État et départements et devant aboutir à la mise sur pied d'un dispositif de mise à l'abri inconditionnelle sur le site, en adéquation avec la situation prévalant dans la zone. Dans une nouvelle décision du 21 juillet 2016, le Défenseur des droits, par ailleurs toujours critique, fait cependant état d'un certain espoir à la perspective de l'ouverture d'un centre dont les contours, fixés à l'été, répondent dans leurs grandes lignes à ses attentes (Décision MDE-2016-186, relative à la situation de mineurs isolés étrangers). Espoir douché, le projet avortant dans la suite du démantèlement de la dernière partie de la jungle (octobre 2016).

Sont alors créés, à la faveur de la circulaire du $1^{\text {er }}$ novembre 2016 relative à la mise en œuvre exceptionnelle d'un dispositif d'orientation des mineurs non accompagnés (sic) dans le cadre des opérations de démantèlement de Calais, les centres d'accueil et d'orientation pour les MIE (CAOMI). Répartis sur l'ensemble du territoire, identifiés par les préfets en concertation avec les collectivités concernées, il est prévu que ces CAO dédiés aux mineurs en accueillent de vingt à cinquante. Ils suscitent cependant de nouveau des réserves de la part du Défenseur des droits, dont il fait état dans le rapport d'observation portant sur le Démantèlement des camps et prise en charge des exilés remis le 20 décembre 2016.

Le Défenseur des droits y conteste, en premier lieu, la légalité même de l'institution de ces CAOMI. Les arguments développés sur ce point par le ministre de la Justice n'emportent pas sa conviction. La question porte ici sur la compétence de l'État pour mettre en place et organiser ce mécanisme de mise à l'abri exceptionnel. Pour sa part, le ministre étaie sa position en prenant appui sur une jurisprudence du Conseil d'État, selon laquelle les " autorités titulaires du pouvoir de police générale " (maire, préfet, Premier ministre) doivent être les garantes de ce que nul ne soit soumis à un traitement inhumain ou dégradant (CE, 27 juillet 2016). Cette situation donnerait alors une base légale à l'intervention de l'État 
lorsque, les départements n'étant plus à même d'assurer efficacement et dans des conditions satisfaisantes la prise en charge des MIE, ces deniers seraient exposés à de tels traitements. Et tel serait précisément le cas dans le Calaisis. Le principe, dans son énoncé, n'est certes guère contestable : de tels traitements ont notamment pu être constatés par le juge administratif, dans les ordonnances déjà citées de novembre 2015. Reste que ce constat, partagé, pouvait, selon le Défenseur des droits, amener d'autres solutions bénéficiant d'une base légale assurée, notamment par une implication financière accrue dans le cadre du dispositif, classique, d'accueil provisoire auprès du Conseil départemental. Le Défenseur des droits ironise d'ailleurs sur l'importance des moyens mobilisés pour ces CAOMI et la célérité avec laquelle ils l'ont été, qu'il compare au manque de mobilisation effective de l'État et du département constatée jusqu'alors, en dépit de ses appels et alertes répétés. À cette illégalité du dispositif s'ajoutent, pour le Défenseur des droits, les conséquences particulièrement dommageables tenant à l'absence de " statut juridique " de mineurs qui seraient " de fait placés dans une zone grise, une zone de non droit ". Aussi bien en appelle-t-il à leur réintégration dans le dispositif de droit commun, autrement plus protecteur. D'autant que le Défenseur des droits relève par ailleurs les nombreuses disparités prévalant au sein des différents CAOMI maillant le territoire.

Exceptionnel en tant qu'il relève intégralement de la compétence (donc du financement) de l'État et place les mineurs hors du droit commun, ce système l'est également par sa finalité. Calais constitue un point de fixation, une étape importante dans les parcours migratoires en raison de sa proximité avec le Royaume-Uni. Nombre de mineurs, tout comme les majeurs, ont pour projet de traverser la Manche, notamment pour y rejoindre des membres de leur famille. Cette situation singulière était initialement envisagée, puisqu'il s'agissait de n'envoyer dans les CAOMI disséminés sur le territoire que les enfants n'ayant pas pour projet de se rendre outre-Manche ou ceux qui, en ayant exprimé le souhait, auraient vu leur demande rejetée par les autorités britanniques. Pour les autres, un accueil devait être organisé au sein du CAP situé dans la Lande de Calais. La circulaire du $1^{\text {er }}$ novembre 2016 revient sur cet engagement. Nulle mention n'y est faite du CAP et, si elle prévoit que l'évaluation de la demande de rapprochement familial au Royaume-Uni doit précéder celle de la situation du mineur au regard de la protection de l'enfance en France, la circulaire n'exclut aucunement que la première soit faite dans le cadre des CAOMI. Tel a été effectivement le cas, puisque dès le 2 novembre, l'ensemble des mineurs présents dans le CAP ont été convoyés vers les CAOMI. Au-delà des conséquences particulièrement fâcheuses de cette situation, qui a fait naître de vives protestations de la part de mineurs ainsi contraints de s'éloigner des côtes britanniques vers lesquelles ils dirigent leurs espoirs et motiver nombre de fugues, sa légalité est discutable. On relèvera à cet égard que, aux fins de rejeter les demandes de suspension des opérations de démantèlement, le Conseil d'État s'était notamment appuyé sur le fait que " [s]'agissant des mineurs isolés, il est précisé que ceux qui souhaitaient aller au Royaume-Uni seront pris en charge, le temps que leur demande soit examinée par les autorités britanniques [...] dans le [CAP] » (CE, ord., 18 oct. 2016).

Enfin, exceptionnel, le dispositif dérogatoire imaginé pour la situation dans le Calaisis, devait n'être que provisoire. II n'en a, ici également, rien été. En décembre 2016, le Défenseur des droits relevait que, sur les soixante-treize 
CAOMI initialement recensés, soixante étaient toujours actifs. Dans une déclaration sur la situation des mineurs isolés placés en CAOMI à l'issue du démantèlement du bidonville de Calais, adopté en Assemblée plénière le 26 janvier 2017, la CNCDH fait pour sa part observer que, sur les 1950 mineurs isolés identifiés sur le site évacué, 860 avaient alors été acceptés par le Royaume-Uni et seuls une cinquantaine avaient bénéficié d'une évaluation sociale. La Commission dresse au-delà un constat particulièrement sévère de la situation, faisant part de son " extrême préoccupation à l'égard des retards pris pour l'évaluation sociale des MIE ", renouvelant " son appel aux autorités locales à intégrer les MIE au plus vite dans le dispositif de droit commun de la protection de l'enfance " et exprimant, " plus généralement ", son inquiétude concernant "la pérennisation de dispositifs spéciaux (CAO et CAOMI), dérogatoires au droit commun, mis en place pour répondre à des situations humanitaires désastreuses consécutives aux carences des pouvoirs publics nationaux et locaux ".

II est alors très significatif que cette déclaration de la CNCDH ait été adoptée le même jour que son avis sur le suivi de l'état d'urgence et les mesures antiterroristes de la loi du 21 juillet 2016. Avis dans lequel elle porte une appréciation particulièrement critique à l'égard d'une situation qui, d'exceptionnelle et provisoire, menace de se banaliser et de se pérenniser.

\section{Références bibliographiques}

Cavaniol Aude (2013) La question épineuse de la répartition territoriale des mineurs isolés étrangers devant le Conseil d'État, Actualité Juridique Droit Administratif, 36, pp. 2107-2111.

Collectif (2016) Quelle " crise migratoire " ?, Plein droit, 111, pp. 3-43.

Debré Isabelle (2010) Les mineurs isolés étrangers en France, Paris, Sénat, 160 p.

Domingo Laurent (2015) Le refus de prise en charge des mineurs isolés étrangers par les départements, La Semaine juridique. Administration et collectivités territoriales, 4.

Lussault Michel (2017) Les hyper-lieux. Les nouvelles géographies de la mondialisation, Paris, Seuil, 320 p.

Senovilla Hernández Daniel (2014) Analyse d'une catégorie juridique récente : le mineur étranger non accompagné, séparé ou isolé, Revue Européenne des Migrations Internationales, 30 (1), pp. 17-34. 